\title{
Minimally invasive transnasal approach for primary ectopic meningioma of the paranasal sinuses
}

\author{
Kornel Szczygielski, Barbara Gałusza, Szczepan Cierniak, Dariusz Jurkiewicz \\ Department of Otorhinolaryngology, Military Institute of Medicine, Warsaw, Poland
}

Videosurgery Miniinv 2015; 10 (3): 477-480

DOI: $10.5114 /$ wiitm.2015.54241

\begin{abstract}
Endoscopic sinus surgery is a standard procedure in the treatment of various pathologies such as chronic sinusitis or some types of neoplasms. The transnasal approach to tumours of paranasal sinuses is favourable due to functional and aesthetic reasons. We report a rare case of a large primary ectopic meningioma of the paranasal sinuses in a 48-year-old woman referred to the Otolaryngology Clinic due to the incidental finding of a pathologic mass visualised on the orthopantomography picture. After diagnosis, the patient was successfully treated with radical transnasal surgery performed under endoscopic vision. In a 1-year follow-up there were no signs of tumour recurrence.
\end{abstract}

Key words: meningioma, paranasal sinuses, endoscopic endonasal approach.

\section{Introduction}

Meningiomas are one of the most common neoplasms of the central nervous system, but in rare cases ( $1 \%$ to $2 \%)$ they are diagnosed in extracranial locations such as the nose and paranasal cavities. Primary ectopic tumours have no direct connection with the cranial cavity. Symptoms are not specific and are correlated with pressure on surrounding structures. The prognosis is usually good because of the benign character of tumour growth. Neoplasms located in the area of the nose and paranasal sinuses should be carefully examined on computed tomography (CT) and magnetic resonance imaging (MRI) scans. The analysis should concern the size, location and expansion of the tumour. When bony gaps to the cranial cavity or orbit occur, it is necessary for the patient to consult a neurosurgeon and ophthalmologist. In most cases of meningiomas the treatment of choice is radical surgery. The transnasal approach to ectopic meningiomas of paranasal sinuses is associated with a shorter period of hospitalisation and a better aesthetic effect [1].

\section{Case report}

The 48-year-old woman was referred to the Otolaryngology Department by the orthodontist due to an incidental, worrying finding in the left maxillary sinus visualised on the orthopantomography picture. The $\mathrm{CT}$ and magnetic resonance imaging revealed an extensive pathologic mass located in the left anterior ethmoid cells, expanding to the nasal cavity, maxillary sinus, modelling the nasal septum and medial orbital wall, as well as involving the middle and upper nasal turbinate (Photos $1 \mathrm{~A}, \mathrm{~B}$ ). After admission of contrast, the mass was enhancing. No intracranial pathologies or bony gaps between the nasal cavity and cranium were described. The patient did not report any problems with breathing through the nose, pathologic discharge from the nose, facial pain or visual disorders. Anterior rhinoscopy revealed normal mucous membrane without evident changes of anatomical structures. In the medical history the patient reported hypothyroidism. She denied exposure to high doses of radiation and hormone therapy in

\section{Address for correspondence}

Barbara Gałusza MD, Department of Otorhinolaryngology, Military Institute of Medicine, 128 Szaserów St, 04-141 Warsaw, Poland,

phone: +48501069 584, e-mail: bgalusza@gmail.com 

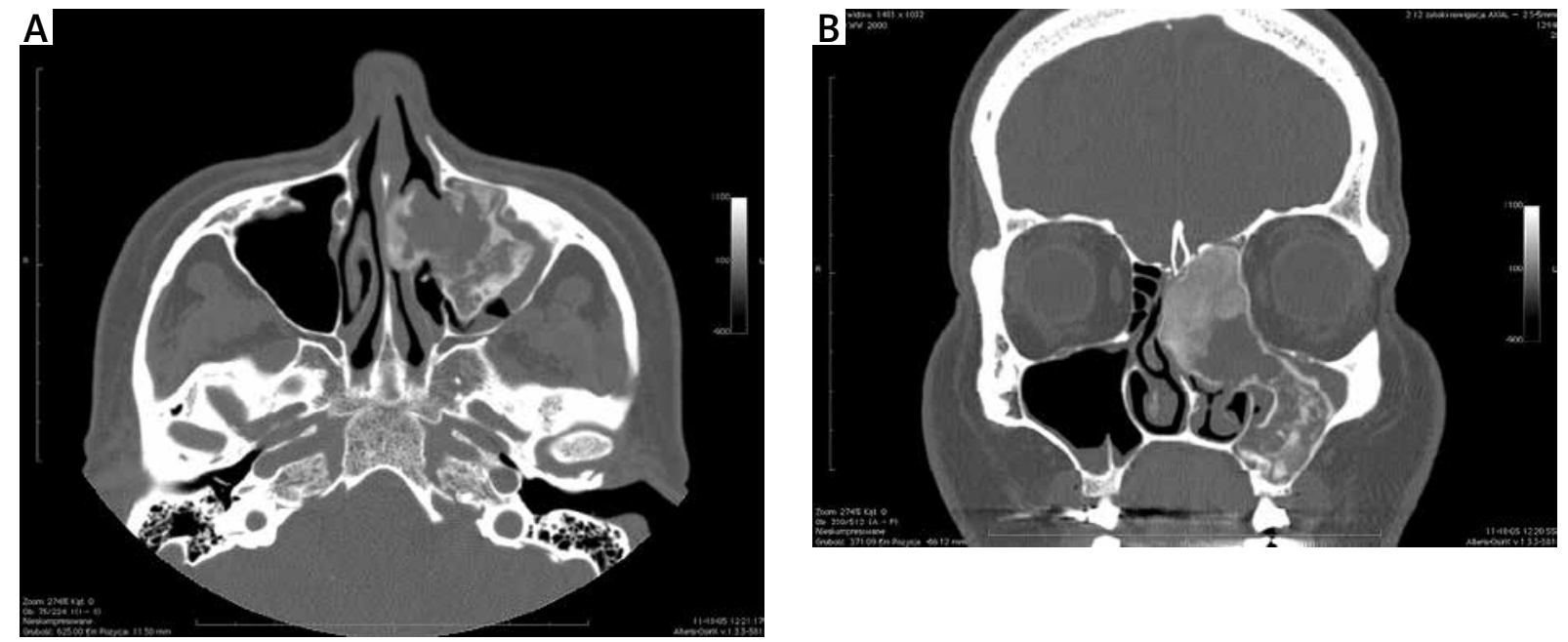

Photo 1. A. Preoperative axial CT scan showing large tumour mass involving left nasal cavity, maxillary and ethmoid sinuses. There is no involvement of the cranial cavity. B - Preoperative coronal CT scan

the past. She has never been treated because of any other tumour and did not have any head trauma.

The biopsy of the tumour was performed under general anaesthesia with endoscopic vision. The histopathological finding was metaplastic meningioma. The immunohistochemical staining revealed co-expression of EMA (+), Vim (+), S-100 (+), and Ki67 (+) (in less than $1 \%$ of tumour cells), which is a typical finding for meningiomas. The patient was qualified for radical tumour removal surgery. The procedure was performed through the nose using $30^{\circ}$ and $70^{\circ}$ endoscopic vision. Medial maxillecto-

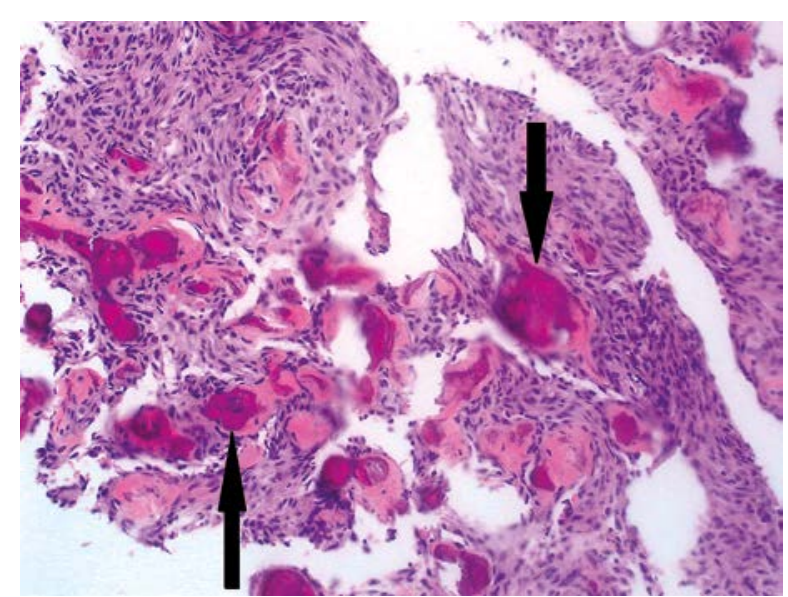

Photo 2. H\&E stain (black and white picture; original magnification 200x). There are numerous calcifications called "psammoma bodies" (indicated by arrows) characteristic for psammomatous meningioma my, ethmoidectomy and total turbinectomy were performed. Ethmoidal roof, lamina papyracea and lateral walls of the maxillary sinus were drilled with a diamond burr. The tumour was removed in pieces. Consistency of the tumour was variable: some of the tissue was spongy in palpation, but some was tough like bone. All the remaining mucous was macroscopically normal. The final pathological diagnosis was psammomatous meningioma (WHO grade I) (Photo 2). Endoscopy of the nose and imaging examinations in the 1-year follow-up did not reveal any signs of tumour recurrence (Photo 3 ). The only patient's complaint after the surgery was excessive nasal discharge from the left nasal cavity due to chronic rhinosinusitis which developed after removal of anatomical structures of the nose. After the treatment with nasal steroid spray the ailment was reduced.

\section{Discussion}

Meningiomas are common neoplasms of the central nervous system but can also be diagnosed in other parts of the body such as the orbit, scalp, nasal cavities and salivary glands as well as on the skin or in the abdomen [2]. Extracranial localisation can be primary or secondary. The secondary extracranial meningiomas grow from the cranium to the surrounding structures. About $20 \%$ of intracranial tumours have a tendency to expand outside of the cranium. This type is more common than primary extracranial meningioma, which does not have any 
direct connection with the cranium. The possible origin of the primary ectopic meningioma is transformation of multipotential mesenchymal cells or Schwann cells into meningocytes or dislocation of embryonic arachnoid cells [3].

Primary ectopic meningioma can be diagnosed when:

- there is no direct connection with the brain and no bony gaps are detected both on radiological scans and during the surgery;

- there is no evidence of intracranial tumour on radiological findings or on the surgical inspection;

- the bony tissue is bulging in the direction of the cranial cavity, not opposite [4].

Histologically there are 15 different kinds of meningiomas, but the most common are meningothelial, fibrous and transitional (mixed). There are no histological differences between intracranial and ectopic meningiomas. Most meningiomas are benign tumours with slow growth, but $2 \%$ to $10 \%$ of them, such as clear cell or papillary type, can have an aggressive clinical course.

Symptoms are not specific and are determined by the location of the tumour. Also radiological findings are not diagnostic. Prognosis is usually good but cannot be defined on the basis of clinical, radiological or even histopathological findings. Recurrence is different, depending on histological type, location, and radicality of the surgery.

In the case of meningioma, computed tomography with contrast or magnetic resonance scans are recommended. Computed tomography helps to determine the correlation between the tumour and bony structures, as well as helping to assess the malignant character of the meningioma when infiltration of the bone is observed. Magnetic resonance imaging shows more clearly the blood flow through the tumour, infiltration, size and relations with surrounding structures.

The mean age of patients with meningiomas is 43.4 years. There are several risk factors that predispose to the growth of meningioma. The first and the most important is exposure to radiation in the past (atomic bombs, often repeated orthopantomograms, radiotherapy) $[5,6]$. Also the influence of hormones is taken into consideration. On some meningiomas receptors to oestrogen, progesterone or androgens can be found. The fact that women are more predisposed to such tumours is significant (55\% in women to $45 \%$ in men). The size of the tumour changes

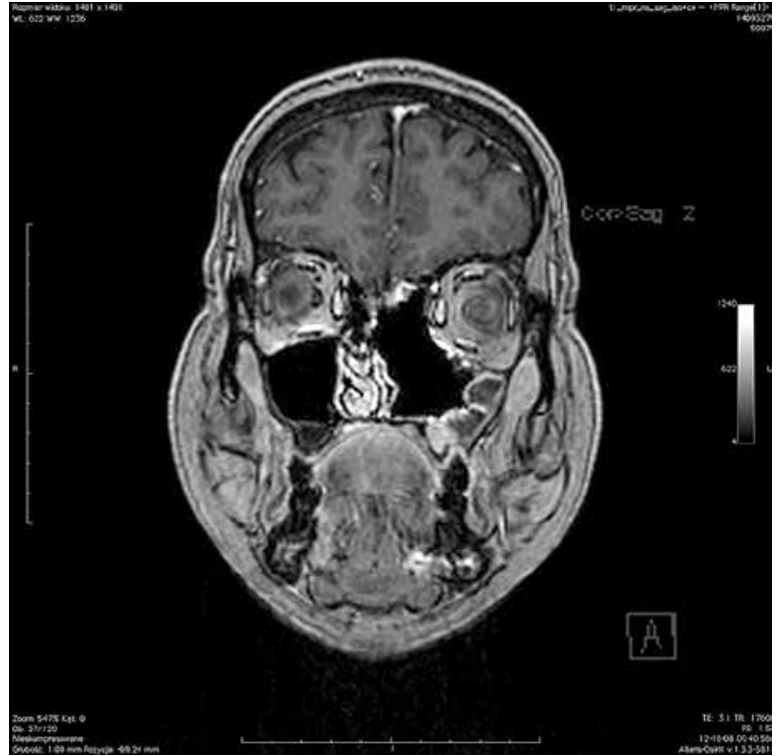

Photo 3. Postoperative MRI scan with no signs of tumour regrowth

during the menstruation cycle or pregnancy. Also a correlation between occurrence of meningiomas and breast cancer is observed. Some studies show that there is a higher risk in the case of admission of hormone replacement therapy or contraceptives. The correlation with cell phone use is still not examined [7].

The treatment of choice in any location is surgery. In some cases, when the surgery is not recommended because of general health problems or in the case of recurrent growth of the tumour, radiotherapy should also be taken into consideration [8].

Endoscopic surgery is successfully applied in the treatment of a wide range of paranasal sinus pathologies. It has become a standard therapeutic procedure not only in patients with rhinosinusitis but also in the case of tumours of the nose and paranasal sinuses such as juvenile angiofibroma or inverted papilloma $[9,10]$. In the last few years we can observe widening scope of indications for endonasal surgery, nowadays including surgery of selected malignancies or surgery of the skull base [11-13]. Also the big size or abundant vascularity of the neoplasm is no longer a contraindication for the transnasal approach. Endoscopic surgery of the nasal and paranasal cavities is favourable to the extranasal approach due to several reasons. When the procedure is performed by an experienced team, the oncological radicality can be achieved at the same 
level as in classical surgery. At the same time, the postoperative morbidity is reduced [13]. For patients it is important that endoscopy provides better functional and aesthetic results. That is why endoscopic surgery is the method of choice in the treatment of the majority of sinonasal tumors.

The authors decided to perform transnasal removal of the large primary ectopic meningioma in volving ethmoidal cells, maxillary sinus, spreading to the nasal cavity, modelling the nasal septum and orbital wall. This method provided oncological radicality, minimal postoperative ailments, and quick convalescence, which resulted in short hospitalisation (the patient was dismissed on the fourth day after surgery) and an excellent aesthetic effect.

\section{Conclusions}

We have presented a case of primary extracranial meningioma located in the nose and paranasal cavities. Primary meningiomas of paranasal sinuses are rare but should be taken into consideration in a differential diagnosis. When bony gaps to the cranial cavity or orbit occur, it is necessary to refer the patient to a neurosurgeon and oculist. Careful preoperative planning allows tumours to be successfully removed with an endoscopic approach.

\section{Conflict of interest}

The authors declare no conflict of interest.

\section{References}

1. Soltys J, Pietniczka-Zaleska M, Mlynczyk-Budzynowska K, et al. Neoplasms of paranasal sinuses in material of ENT Department MSS Hospital in Warsaw between 2006-2007. Otolaryngol Pol 2008; 62: 451-4.

2. Iaconetta G, Santella A, Friscia M, et al. Extracranial primary and secondary meningiomas. Int J Oral Maxillofac Surg 2012; 41: 211-7.

3. Yamashima T, Kida S, Yamamoto S. Ultrastructual comparison of arachnoid villi and meningiomas in man. Mod Pathol 1988; 1: 224-34.

4. Daneshi A, Asghari A, Bahramy E. Primary meningioma of the ethmoid sinus: a case report. Ear Nose Throat I 2003; 82: 310-1.

5. Preston DL, Ron E, Yonehara S, et al. Tumors of the nervous system and pituitary gland associated with atomic bomb radiation exposure. J Natl Cancer Inst 2002; 94: 1555-63.

6. Ron E, Modan B, Boice JD Jr, et al. Tumors of the brain and nervous system after radiotherapy in childhood. N Engl J Med 1988; 319: 1033-9.

7. Wiemels J, Wrensch M, Claus EB. Epidemiology and etiology of meningioma. J Neurooncol 2010; 99: 307-14.
8. Yamada SM, Yamada S, Takahashi H, et al. Extracranially extended meningothelial meningiomas with a high MIB-1 index: a report of two cases. Neuropathology 2004; 24: 66-71.

9. Lombardi D, Tomenzoli D, Butta L, et al. Limitations and complications of endoscopic surgery for treatment for sinonasal inverted papilloma: a re-assessment after 212 cases. Head Neck 2011; 33: 1154-61.

10. Busquets JM, Hwang PH. Endoscopic resection of sinonasal inverted papilloma: a meta-analysis. Otolaryngol Head Neck Surg 2006; 134: 476-82.

11. Gotlib T, Osuch-Wojcikiewicz E, Held-Ziolkowska M, et al. Endoscopic transnasal management of sinonasal malignancies our initial experience. Videosurgery Miniinv 2014; 9: 131-7.

12. Saedi, B, Aghili M, Motiee M, et al. Surgical outcomes of malignant sinonasal tumours: open versus endoscopic surgical approaches. J Laryngol Otol 2014; 128: 784-90.

13. Lund V, Stamberger H, Nicolai P, et al. European position paper on endoscopic management of tumors of the nose and paranasal sinuses and skull base. Rhinology 2010; Suppl. 22: 1-143.

Received: 25.05.2015, accepted: 19.08.2015. 